\title{
Geschlossene, offene oder gar keine Grenzen? Zur Legitimität von (Staats-)Grenzen
}

\section{Christian Banse}

\begin{abstract}
Wie und warum werden Grenzen von Nationalstaaten legitimiert? Nach sozial- und grenzwissenschaftlichem Forschungsstand, der noch kaum auf empirischen Ergebnissen basiert, sind Grenzen Ort oder Gegenstand von Konflikten. Fragen zur Legitimität beziehen sich auf staatliche und supranationale Grenzpolitik gegenüber Migrant*innen und auf Grenzveränderungen in Grenzregionen. Grenzen polarisieren, weil sie Unterscheidungen und Gegensätze etablieren und symbolisieren. Ihre Legitimität steht infrage, gerade wenn verschiedene Perspektiven berücksichtigt werden. Die moralische Dimension von Grenzziehungen tritt in den Vordergrund.
\end{abstract}

\section{Schlagwörter}

Staatsgrenze, Legitimität, Rechtfertigung, Grenzbegriff

\section{Einleitung}

Fragen zur Legitimität staatlicher Institutionen und Handlungen sind meist im politik- und rechtswissenschaftlichen, manchmal auch im philosophischen Bereich verortet und beziehen sich in der Regel auf die Rechtmäßigkeit einer politischen Ordnung. Legitim ist eine Ordnung dann, wenn sie durch bestimmte festgelegte, demokratische Verfahren von den an dieser Ordnung Beteiligten anerkannt wird und als Norm gelten kann (vgl. Forst 2015, S. 186ff.). Die Norm muss zumindest in der politischen Form der Demokratie den an dieser Ordnung Beteiligten gegenüber gerechtfertigt und begründet werden können (vgl. ebd., S. 190) und basiert auf einem allgemeinen moralischen Grundanspruch, der dem Selbstverständnis demokratischer Staaten entspricht: Man könnte der Norm als freie und gleiche Person zustimmen. Fragen zur Legitimität gehen in diesem Verständnis also immer mit potenziellen Rechtfertigungen einher, die auf einem gemeinsamen normativen Grund basieren (vgl. Dammayr et al. 2015, S. 7). ${ }^{1}$ Im Folgenden werden diese politisch-rechtlichen Begründungen als normative Legitimität bezeichnet.

„Legitimationsprobleme“ (Habermas 1971), die im Sinne politisch-rechtlicher Begründungen im Zusammenhang mit den territorialen Grenzen des Hoheitsgebiets des souveränen Staates gesehen werden, beziehen sich in der Regel auf die Rechtmäßigkeit staatlichen Handelns an seinen Grenzen, etwa gegenüber dem Nachbarstaat, der Staatengemeinschaft oder vor allem gegenüber dem Handeln von Akteuren, die nicht Mitglied des Staates sind, wie etwa Migrant*innen und Geflüchtete. Die Legitimität wird zumeist dann ein Gegenstand der öffentlichen Auseinandersetzung, wenn eine Krise, etwa die sogenannte Flüchtlingskrise 2015, festgestellt wird (vgl. Dammayr et al. 2015, S. 7f.). Um Legitimationsprobleme, die auf eine normative Krise der nationalen Grenze hinweisen, soll es hier gehen.

1 Dieser ,Grund“ wird im Alltag, wie Albrecht Koschorke (2017, S. 158f.) betont, selten umfassend gerechtfertigt. Nötige Legitimierungen werden von Deutungseliten und Legitimierungsexpert*innen übernommen. 
Bei der Analyse der normativen Legitimität von Grenzziehungen heute zeigt sich jedoch ein grundlegendes Problem, das eine wissenschaftliche Auseinandersetzung mit diesem Thema erschwert: Geradezu offensichtlich beanspruchen nationale Grenzen Legitimität. Sie sind der sichtbare Beleg dieses Anspruchs. Wer eine Grenze zieht oder seine Grenze schützt, glaubt sich als staatliche, souveräne (und damit legitimierte) Macht im Recht; schon ihr faktisches Vorhandensein scheint die Grenze zu legitimieren (vgl. Falk 2011, S. 9f.). ${ }^{2}$ Aus der Sicht des grenzziehenden Staates also ist die Frage nach der Legitimität seiner Grenzen beantwortet. Nationale Grenzen werden so gleichsam naturalisiert und müssen gar nicht gerechtfertigt werden (vgl. Schultz 1993). Man könnte auch sagen: Wer seine Grenzen nicht kontrollieren kann, ist kein souveräner Staat (vgl. Herrmann/Vasilache in diesem Band). Positionen, die die normative Legitimität einer Grenzziehung hinterfragen, haben oft Probleme, einen anerkannten Bezugspunkt zu finden, wie die Vielzahl von Grenzveränderungen und -überschreitungen im Europa des 20. Jahrhunderts zeigen (vgl. Bös/Zimmer 2006).

Diese vermeintliche Evidenz der Staatsgrenze wirft allerdings Fragen auf, sobald man weitere Akteure, die eigene Grenzen ziehen bzw. vorgefundene nicht akzeptieren oder überschreiten, in die Grenzbeobachtung einbezieht. ${ }^{3}$ Grenzen sind weitaus weniger selbstverständlich, wenn die soziale Herstellung der Legitimität der Grenze berücksichtigt wird. Dabei geht es um die Anerkennung sozialer Ordnung, die über die politisch-rechtliche Legitimation von Grenzen hinausgeht und das Handeln von Akteuren, die mit der Grenze konfrontiert sind, in den Blick nimmt. ${ }^{4}$ Aus einer soziologischen Perspektive sind Staatsgrenzen keinesfalls selbstverständlich. Soziologisch wird seit einiger Zeit die Legitimität im Zusammenhang mit Grenzen dann zum Thema, wenn es um weitreichende gesellschaftliche Veränderungen geht, die makrosoziologische Theorien diagnostizieren (Mann 1997; Beck 1999; Mau 2006; Vobruba 2006). Ein gesellschaftlicher Wandel durch Phänomene globaler Reichweite (wirtschaftliche Globalisierung, Umweltprobleme, weltweite Migration, mediale Vernetzung und Pandemien) sorgt für Einschätzungen, in denen nach der Legitimität staatlicher Grenzpolitik gefragt wird. Vor diesem Hintergrund unterliegen heutige Grenzpraktiken und Grenzziehungen einem normativen Legitimierungsdruck, mit dem Staaten und Akteure der Grenzregime sich gegenüber einer kritischen (sozialwissenschaftlich fundierten) Öffentlichkeit auseinandersetzen müssen. Von Sozial-, insbesondere Grenz- und Migrationsforscher*innen wird dementsprechend - allerdings meist implizit - gefragt: Wie und warum werden nationale Grenzen und ihre teils unmenschlichen Effekte legitimiert, wenn in der heutigen ,globalisierten' und vernetzten Welt grenzüberschreitende Prozesse und Interessen zunehmen? Wie wird mit der Tatsache umge-

2 In diesem Zusammenhang wäre heute eine Analyse der Visualisierungen von nationalen Grenzen wichtig. Francesca Falk (2011) liefert erste Gedanken dazu. Georg Vobruba (2006) betont die medial erzeugten Bilder von Grenzen, die uns vertraut sind.

3 Zur soziologischen Beobachtung von Grenzen vgl. Vobruba (2006). Er stellt eine Akteursperspektive der (staatlichen) Steuerungsperspektive gegenüber. Grenzen sind zum einen Steuerungsinstrumente, die etwa den Grenzübertritt regeln, zum anderen aber werden durch Grenzen auch Handlungschancen für diverse Akteure generiert, die unterschiedliche Interessen haben.

4 Vgl. zum soziologischen Begriff der Legitimität Max Weber (1921/1980, S. 16f.), der Verbindlichkeit als ein Element erläutert. Hier schließen dann Theorien der Legitimität an, die von ,ideologischen “ Formen der Rechtfertigung handeln. Jürgen Habermas etwa sieht „Legitimationsprobleme im Spätkapitalismus“ (1971), Pierre Bourdieu (2014) verdeutlicht, wie sehr dem Legitimierungsbedürftigen eine als selbstverständlich anerkannte symbolische Gewalt zugrunde liegt, und für Niklas Luhmann (2000) ist vor allem die Staatsgewalt als sich selbst legitimierende Instanz legitimierungsbedürftig. Einen Überblick über die sozialwissenschaftliche Beschäftigung mit Legitimität findet sich bei Maria Dammayr et al. (2015). Besonders die Entstehung von „Rechtfertigungssemantiken“ (ebd., S. 7) steht dabei im Vordergrund. Vgl. auch einschlägig Luc Boltanski und Laurent Thévenot (2007) zur Pluralität legitimer Rechtfertigungen. 
gangen, dass an den nationalen und supranationalen Grenzen Menschen um ihr Überleben kämpfen und auch sterben? Welche Grenzüberschreitungen können legitim sein? Wie wird heute die geltende staatliche Norm im Zusammenhang mit nationalen Grenzziehungen und ihren Effekten begründet oder hinterfragt? Die grenzsoziologische Betrachtung zur Legitimität nationaler Grenzen, die in diesem Beitrag nachvollzogen werden soll, befragt die Forschung danach, wie und warum Grenzen legitimiert werden. Dazu muss die empirische Beobachtung von Handlungen, die direkt auf die nationale Grenze hin stattfinden, in die Überlegungen zu ihrer Legitimität einbezogen werden. Im Folgenden wird in analytischer Abgrenzung zur politisch-rechtlich fundierten normativen Legitimität der Grenze von einer empirischen Legitimität gesprochen, wenn es um Rechtfertigungen eines Handelns in Bezug zur nationalen Grenze geht, das an den Grenzen beobachtet werden kann. ${ }^{5}$

Der folgende Beitrag widmet sich also der Legitimität von nationalen Grenzen nicht allein aus der normativen Perspektive staatlicher Akteure, sondern auch aus einer sozialwissenschaftlichen, meist empirischen Perspektive, die das Zusammenspiel verschiedener Akteure und Gruppen in ihrer jeweiligen Dynamik untersucht. Wie jede Wissenschaft muss auch die Grenzsoziologie ihre Perspektive auf ihren Gegenstand reflektieren. Eine grenzsoziologische Bewertung von Grenzen, etwa wenn sie Effekte von Grenzregimen offenlegt und kritisiert, ist selbst Teil des Geschehens - Wissenschaft ist hier ein Akteur, der ebenfalls ,beobachtet` werden muss bzw. der sich selbst beobachten muss (vgl. Luhmann 1982; Vobruba 2006). Auch wenn alltagspolitisch ein hohes Diskussionsaufkommen besteht, was das Für und Wider von etwa exkludierenden Grenzpraktiken oder der Grenze selbst betrifft, so wird in der empirischen Erforschung von nationalen Grenzen noch wenig systematisch reflektiert, wie und warum gerade Grenzen Gegenstand von gegensätzlichen normativen Rechtfertigungen werden und wie dem Normativen von Grenzen forschungspraktisch angemessen beizukommen ist.

Ausgehend vom Ziel einer systematischen Erörterung des Verhältnisses von Grenzen und Legitimität gilt es in diesem Beitrag zunächst zu verstehen, wie bzw. vor welchem Hintergrund die Grenzforschung ihre spezifische Perspektive entwickelt hat. Dazu muss man wissen, in welcher Tradition die normative Legitimität von Grenzen und Grenzüberschreitungen sozialwissenschaftlich fokussiert werden kann. Daran anschließend kann an den für die Grenzforschung einschlägigen Forschungsfeldern Migration und Grenzregionen gezeigt werden, dass die Forschung mit kontroversen und diversen empirischen Legitimierungsprozessen zu tun hat, die auch etwas über das normative Verständnis von nationalen Grenzen aussagen. Der Beitrag schließt mit den Fragen, wie eine sozialwissenschaftliche Perspektive auf Legitimationen von (nationalen) Grenzen in der Gesellschaft heute aussehen könnte und warum sie gesellschaftlich wichtig ist.

5 Das Begriffspaar normative Legitimität/empirische Legitimität soll einen Gegensatz idealtypisch verdeutlichen: Während normative Legitimität meint, dass auf einen allgemein politisch-rechtlich anerkannten und herrschenden Rahmen in der Rechtfertigung (oder der Kritik) Bezug genommen wird, betont empirische Legitimität die konkrete Praxis und deren Rechtfertigung. Beide sind aus soziologischer Sicht eng verbunden, weil die normative Legitimität Praxis werden muss (es gibt ja Grenzen, die empirisch feststellbar normativ sind, weil Akteure sie mit Nachdruck ziehen) und weil die empirische (alltägliche) Legitimität auf Normen Bezug nehmen oder selbst normativ werden kann. Die normative Dimension der Legitimität ist eine Setzung, die empirisch bestätigt oder hinterfragt werden kann und die eine mögliche Abweichung bzw. Grenzüberschreitung verhindern soll (vgl. Kaufmann et al. 2002, S. 8). 


\section{Die Legitimität der Grenzforschung}

\subsection{Eine kleine Geschichte der (De-)Legitimierung von Grenzen}

Für die Darstellung der Zugänge der Grenzforschung zur Legitimität von (Staats-)Grenzen muss gefragt werden, welche gesellschaftlichen Dynamiken der wissenschaftlichen Konzeptualisierung von Grenzen zugrunde liegen, die für die Frage nach ihrer Legitimität relevant sein könnten. Eine sozialwissenschaftliche Perspektive auf die heutige Legitimität von nationalstaatlichen Grenzen muss den sozialhistorischen Kontext berücksichtigen, der zur wissenschaftlichen Beschäftigung mit Grenzen geführt hat. Die Frage nach der Legitimität von Staatsgrenzen heute ist ohne die widersprüchliche gesellschaftliche Entwicklung der letzten 30 Jahre nicht zu denken (vgl. Newman/Paasi 1998; Banse 2013; Graziano 2018; Paasi 2019). Die Konzepte von Grenzen, ihre metaphorischen Bezüge und impliziten Bewertungen, die in der wissenschaftlichen Reflexion von Grenzen zu finden sind, sind mit politischen Entwicklungen verwoben (vgl. dazu Schmieder in diesem Band), deren Berücksichtigung verständlich machen kann, warum die Frage nach der Legitimität von Grenzziehungen forschungspraktisch relevant wird.

Grenzforschung, als interdisziplinäre Antwort auf empirische, methodische und gesellschaftspolitische Fragen der 1990er-Jahre, hat sich im Zuge des seit dem Fall des Eisernen Vorhangs stattfindenden gesellschaftlichen Wandels mit verschiedenen Auflösungen von nationalen Grenzen beschäftigen müssen und dabei Grenzen neu bewertet (vgl. Baud/Schendel 1997; Newman/Paasi 1998; Yarbrough/Yarbrough 2003). Bis dahin waren sie zwar ein Forschungsgegenstand der Geografie, Rechts- und Geschichtswissenschaft, wurden aber in der Regel als vorzufindende Tatsache behandelt. Grenzen waren Ausdruck des Souveränitätsprinzips von sich selbst bestimmenden Nationalstaaten, wie es zuerst im Westfälischen Frieden formuliert wurde (vgl. Graziano 2018, S. 14-17). Für das internationale Recht sind Grenzlinien, die den politischen Raum definieren, weiterhin grundlegend (vgl. ebd., S. 9f.; Paasi 2019). Die Bildung neuer Staatengemeinschaften und politischer Bündnisse auch im Zusammenhang mit Sicherheitsfragen bezüglich terroristischer Bedrohungen haben jedoch das alte Verständnis von Souveränität relativiert. In den Border Studies (Gerst et al. 2018), der empirischen Forschung zu Grenzregionen (Velde/Houtum 2000) und diversen fachspezifischen Forschungen wie etwa der Europasoziologie (Bach 2010), der Analyse der Globalisierung (Beck 1999) bzw. der Weltgesellschaft (Schimank 2005) und der Raumsoziologie (Schroer 2006) geht es seitdem um die Frage nach der Legitimität politisch-territorialer Grenzen des modernen Nationalstaats. Gefragt wird, ob sich Grenzen zunehmend auflösen oder ob sie als soziale Grenzphänomene (social boundaries; Fassin 2020) substituiert werden. Ob Grenzen zunehmend verschwinden oder sich verlagern (de-/rebordering), kann dementsprechend als grundlegende Frage der zeitgenössischen Grenzforschung gelten, die sich vor allem dadurch im Wissenschaftsbetrieb legitimiert, dass sie den Prozess der Entgrenzung sowie die Rückkehr oder Neubildung von verschiedenen Grenzpraktiken beobachtet und bewertet.

Aus diesem Kontext heraus hat sich nicht nur die Perspektive auf die Grenze verändert, die vorher vor allem als die Nationalstaaten trennende Linie, häufig auch als kaum zu überwindende Block- und Systemgrenze gesehen wurde, einschlägig repräsentiert durch die Berliner Mauer. Verändert haben sich auch der Begriff der Grenze selbst und dementsprechend sei- 
ne forschungspraktischen Konzeptualisierungen, die mit einem viel tieferen Verständnis der Funktionen und Dimensionen von Grenzen operieren (vgl. Fassin 2020, S. 6f.). Inwieweit die verwendeten Begriffe, theoretischen Konzepte und Forschungsmethoden der sich mit Grenzen beschäftigenden Forschungsbereiche differieren oder sich in ihren Bedeutungen überschneiden, kann hier nicht ausgeführt werden (siehe dazu Gerst/Krämer in diesem Band).

Gegen die als „methodologischen Nationalismus“ (Beck 1999, S. 49f.) bezeichnete, lange vorherrschende Engführung von Grenzen als Endpunkt gesellschaftlicher Zusammenhänge (vgl. Banse 2013, S. 28f.) wurde zunächst mit Emphase die zunehmende Grenzöffnung und weltweite Vernetzung in einer globalen Welt (borderless world) festgestellt; deren mögliche Folgen wurden begrüßt (Ohmae 1990; Paasi 2019). Vor dem Hintergrund beispielloser Grenzöffnungen in Europa und anderen Regionen der Welt wurde vor allem aus sozialwissenschaftlicher Perspektive jede Grenzziehung - besonders, wenn sie räumlich und territorial umgesetzt wurde - rechtfertigungsbedürftig. In Ansätzen, die im Austausch mit Integrationsprogrammen der Europäischen Union entstanden sind, wurden die Grenzüberschreitungen und die institutionalisierte grenzüberschreitende Zusammenarbeit, die von der Politik gefördert wurde, wichtig (Bach 2006; Mau 2006). Besonders Grenzregionen wurden für die wissenschaftliche Beobachtung von Grenzphänomenen ein Gegenstand, an dem sich eine enge Verknüpfung von Forschung und Politik realisierte: Grenzen wurden in den politischen Leitlinien von Trennungslinien zu Kontaktzonen umdefiniert, in denen es neue kulturelle und soziale Phänomene zu entdecken gab, die u.a. Forschungen aus Kultur- und Sozialwissenschaften bereicherten. Vor allem wurde konzeptuell die Durchlässigkeit von Grenzen betont und als Chance für Begegnungen, Austausch und wirtschaftliche Innovationen bewertet - gerade wenn man sich exemplarisch die europäische Politik der (Grenz-)Regionen vergegenwärtigt (vgl. Banse 2013, S. 85-92). In diesem Zusammenhang sind Grenzüberschreitungen und ihre Erforschung in einem politischen Rahmen legitimiert (und rechtlich kodifiziert) worden, dem es um die Überwindung der europäischen Grenzlinien als „Narben der Geschichte“ (AGEG 2008, S. 15) ging, also der Trennlinien, die im Zusammenhang mit verschiedenen Kriegen entstanden sind. Grenzüberschreitende Zusammenarbeit ging einher mit einer politischen Legitimierung von Grenzen als Begegnungsort, wo es im besten Fall zur Auflösung der Barrieren kommen würde, sodass dementsprechend Grenzen als Barrieren - zumindest im sozialwissenschaftlich inspirierten Diskurs von europäischen Institutionen ${ }^{6}$ - delegitimiert wurden. ${ }^{7}$

In der Folge der Wahrnehmung ,neuer' Grenzphänomene, die paradoxerweise mit der Europäisierung, Globalisierung und durch die technischen Möglichkeiten der Vernetzung erst in ihrem Ausmaß sichtbar wurden oder sogar entstanden sind (vgl. Bach 2006; Schroer 2006; Banse 2013), wurden Grenzen als Gegenstand der nun sich verstärkt etablierenden Grenzforschung methodologisch zunehmend weniger als rein räumliches bzw. territoriales, sondern als ein multidimensionales und der vielfältigen Effekte wegen komplexes Phänomen eingeschätzt (vgl. auch Wille in diesem Band). Die Staatsgrenze etwa wird seitdem multiperspektivisch von unterschiedlichen Disziplinen wie der Politischen Geografie, den Rechts-, Politik-, Geschichts-, Kultur- und Sozialwissenschaften (und häufig auch als ästhetisches Phänomen) in den Blick genommen. Nachhaltige begriffliche Übereinstimmungen, auch innerhalb der Sozialwissenschaf-

6 Zur Frage nach der normativen Legitimität von Grenzen würde auch die demokratische Legitimierung der europäischen Politik insgesamt gehören, auf die hier nicht eingegangen wird (vgl. dazu aber Dux 2010).

7 In diesen Diskurs der Delegitimierung gehört die neoliberale Einschätzung, die offene Grenzen favorisiert (vgl. Paasi 2019). Trotzdem kommen auch liberale Modelle offener Grenzen in der EU nicht ohne eine Verschiebung von ,harten' Grenzmaßnahmen vom Nationalstaat zur europäischen Außengrenze aus (vgl. Banse et al. 2007). 
ten, die Grenzen und Grenzziehungsprozesse methodisch recht divers untersuchen, bleiben noch aus. Auf jeden Fall bezeugen die Forschungen die besondere Bedeutung, die Grenzen heute kulturell und sozial zugesprochen wird.

Diese neu entdeckte Multifunktionalität und Mehrdimensionalität von Grenzen beeinflusst auch die wissenschaftliche Beschäftigung mit der Frage der Legitimität von Staatsgrenzen. Vor dem Hintergrund der konzeptionellen und disziplinären Ausdifferenzierung der Grenzforschung werden ganz unterschiedliche Legitimationserzählungen möglich, je nachdem, ob man die Legalität der Grenzübertritte bzw. des Grenzregimehandelns, Humanität als ethischen Maßstab oder etwa die historische Rechtfertigung von Grenzziehungen fokussiert. Die Ausweitung des Grenzbegriffs verlangt, dass die Grenzforschung Grenzen im jeweiligen empirischen Kontext beobachtet. Die Vielzahl an Akteuren, die Grenzen legitimieren oder delegitimieren, können sich in ihren Zielen widersprechen, verschiedene Aspekte der Grenze ansprechen und gegensätzliche Interessen verfolgen.

\subsection{Die Komplexität von Grenzlegitimierungen und ihrer empirischen Erforschung}

Grenzforschung als sozialwissenschaftliche, interdisziplinäre Perspektive rechtfertigt sich heute vor allem durch eine Herangehensweise, die der Komplexität ihres Gegenstandes gerecht wird. Aus der Sicht der Forschung ist die Komplexität der nationalen Grenze ein Spiegelbild der aktuellen politischen Anforderungen einer globalisierten und zugleich nationalstaatlich organisierten Welt, in der die Frage nach der legitimen Funktion nationaler Grenzen gestellt wird (vgl. Schimank 2005).

Wenn es um die empirische Erforschung von Legitimierungen nationaler Grenzen geht, muss man davon ausgehen, dass sich die hoffnungsvollen Vorstellungen zur Auflösung der nationalen Grenze, wie sozialwissenschaftliche Theorien und politische Leitlinien einst annahmen, nicht erfüllten. Die Staatsgrenze ist weiterhin weltweit ein normatives Instrument, mit dem politische Interessen reguliert werden. Gleichzeitig können sich offizielle normative Legitimitätsvorstellungen von den Legitimitätsvorstellungen anderer Akteure an der Grenze empirisch massiv unterscheiden (vgl. Heyman/Symons 2015). Dementsprechend müsste jeder sozialwissenschaftlichen Befragung der normativen Legitimität einer nationalen Grenze die politische Vorstellung der Grenze und ihre empirische Entsprechung gegenübergestellt werden. Jede politische Vorstellung von Grenze drückt zunächst eine normative (inkludierende und exkludierende) Vorstellung der staatlichen Akteure aus; ihre empirische Realität weicht jedoch von dieser Vorstellung ab. So werden Grenzen normativ als undurchlässige Schutzwälle imaginiert, während aus empirischer Sicht Grenzen durchlässig sind (Migration), oder als durchlässig deklariert, obwohl sie immer noch in bestimmten Aspekten trennen (Grenzregionen). Grenzen sind deshalb immer auch ein Mittel symbolischer Politik. Sozialwissenschaftlich muss also die Erforschung von nationalen Grenzen und ihrer Legitimität von einer Differenz von empirischer Realität und ,normierender' Symbolik ausgehen und die staatliche Akteur*innenperspektive hinterfragen.

Dazu kommt, dass es nicht nur eine Differenz von Symbolpolitik und Grenzempirie gibt, sondern dass man unterschiedliche Realitäten an Grenzen und damit unterschiedliche Grenzerfahrungen feststellen kann. Das hat zum großen Teil damit zu tun, dass Grenzen sich für die, die von ihnen betroffen sind, ganz unterschiedlich darstellen können, je nachdem, welche 
Funktion der Grenzübertritt für die Grenzgänger*innen hat und mit welchem Hintergrund er ausgeführt bzw. verhindert wird (vgl. Vobruba 2006; Graziano 2018).

Die Akteur*innen an der nationalen Grenze sind nicht nur unterschiedlich von ihr betroffen, sondern sie bewerten sie zuweilen gegensätzlich. Die Grenze ist von Interpretationskonflikten geprägt; die Analyse sollte deren Nachvollzug, die sich auch in antagonistischen Vorstellungen von der Legitimität der gezogenen Grenze ausdrücken können, ermöglichen. Es könnte also durchaus kontextabhängig sein, was für Protagonist*innen der Grenze legitim ist. Mit diesen Gegensätzen sind auch moralische Einschätzungen, die den Übertritt je nach Akteur und Herkunft bewerten, verbunden: Von undokumentierter Migration etwa profitieren Unternehmer*innen vermutlich mehr als einheimische Arbeiter*innen, die Billiglohnkonkurrenz fürchten müssen.

Während es bisher um eine Dialektik von gesellschaftspolitischem Wandel und Forschung ${ }^{8}$ ging, in der der historische und konzeptuelle Ort, von dem aus nach der normativen Legitimität von Grenzen gefragt wird, thematisiert wurde, steht nun die Legitimität der nationalen Grenze als empirischer Gegenstand selbst im Vordergrund. Legitimationsprobleme von Grenzen werden an zwei exemplarischen Gegenständen - Migration und Grenzregionen - dargestellt, die auf unterschiedliche Weise die Ambivalenz von Grenzen und ihren Rechtfertigungen zeigen.

\section{Die Erforschung der Grenzlegitimität}

\subsection{Legitimationsprobleme von Migration und Grenzpolitik}

Die Frage nach der Legitimität wird, wie eingangs betont, meist dann aufgeworfen, wenn Krisen festgestellt werden. Im Zusammenhang mit nationalen Grenzen werden vor allem die unterschiedlichen Lebenschancen thematisiert, die von staatlichen und supranationalen Grenzregimen beeinflusst, ermöglicht oder verhindert werden (vgl. Vobruba 2006). Besondere Aufmerksamkeit bekommt die Bewertung von Migration und Flucht über Staatsgrenzen hinweg, also die Frage nach der Legitimität von transnationalen Grenzüberschreitungen und von Grenzschließungen von Staaten und Staatenbündnissen (vgl. Brown 2018). Im öffentlichen Diskurs dominieren Ansätze, die dem staatlichen (oder supranationalen) Souveränitätsgedanken verpflichtet sind (vgl. Heyman/Symons 2015).

Eine nationale Herleitung der Grenzen kann auf eine lange Tradition von Rechtfertigungen zurückgreifen (vgl. Bielefeld 2003). ${ }^{9}$ Sie steht vor allem mit der modernen Definition des Staates im Zusammenhang, der sein Gewaltmonopol mit dem klar eingegrenzten Staatsgebiet, in dem die Bevölkerung lebt, verknüpft (vgl. Flügel-Martinsen et al. 2018). In der Sichtweise des souveränen Nationalstaats stehen die Interessen des jeweiligen Staates geradezu selbstverständlich im Vordergrund: Begründungen von Exklusionsmaßnahmen lassen sich auf das Selbstbestimmungsrecht der Nation beziehen und ihre populäre Darstellung auf Landkarten zeigt klare Trennlinien, die Territorien unterscheiden und eine explizite Differenz zwischen dem Eigenen und dem Anderen implizieren (vgl. Bielefeld 2003). Der Vorteil einer so konzeptionierten

8 Ich danke Maria Klessmann für diese zusammenfassende Formulierung.

9 Einen Überblick über die philosophischen Grenztheorien von Locke, Hobbes und Rousseau findet sich bei Andreas Vasilache (2007). 
Grenze ist ihre Eindeutigkeit (vgl. Luhmann 1982). Als rechtfertigende Erzählung fungiert in dieser Tradition ein nationales Identitätsnarrativ, das lange Zeit im Kontext der nationalstaatlichen Verfassung der Welt als Norm gelten konnte. In seiner aktuellen Variante ist das Narrativ oft von artikulierten Ängsten und Warnungen zu grenzüberschreitenden Phänomenen, etwa Terrorismus, Epidemien und vor allem Migration, bestimmt, vor der die geschlossene nationale Grenze schützen soll. Exkludierende bzw. inkludierende Grenzpolitik findet vor diesem Hintergrund als Bestandteil eines als notwendig angesehenen Sicherheitsdispositivs, das den Schutz der Bevölkerung in den Vordergrund stellt, ihre Legitimation (vgl. Falk 2011). Als normative oder moralisierende Grundlage kann die Bevorzugung der eigenen Gruppe gelten, die sich in diesem Konzept als eine Selbstverständlichkeit darstellt. Das Selbstbestimmungsrecht einer Nation wird als Begründung herangezogen. Scharf kontrollierende Grenzregime wie an der US-amerikanisch-mexikanischen Grenze, an der illegale Grenzübertritte bekämpft werden und Verhaftungen in diesem Zusammenhang als Erfolg gelten, können der symbolische Ausdruck dieses Konzepts sein (vgl. Brown 2018, S. 67).

Besonders die vielfach festgestellte Rückkehr von Mauern und Zäunen kann als räumliche Manifestation des souveränen Anspruchs und als symbolische Politik gelten (vgl. ebd.; siehe auch Leuenberger in diesem Band). Als Reaktion auf die erwähnten Entgrenzungsprozesse wird durch den Staat eine Notwendigkeit der Grenzen behauptet. Wendy Brown betont die Legitimitätsdefizite, die die neuen Grenzbefestigungen weltweit betreffen, und stellt trotz aller regionalen Unterschiedlichkeit spezifischer Rechtfertigungen fest, dass weiterhin auf die „Idee nationalstaatlicher Souveränität“ (ebd., S. 59) rekurriert wird, die sozusagen performativ von der Mauer unterstützt wird. In diesem Zusammenhang ist häufig von einem „temporären Charakter der neuen Mauern“ (ebd., S. 60) die Rede, der durch den Bezug auf einen Notstand gerechtfertigt wird. Für Brown zeigen sich in der spektakelhaften Inszenierung des Grenzregimes eines souveränen Nationalstaats Legitimationsstrategien, die auf fast paradoxe Weise anzeigen, wie sehr Grenzen eher als postsouverän verstanden werden sollten.

Brown diskutiert allerdings nur die eine Seite der Legitimationsdiskurse, die Grenzen als notwendiges Instrument des Schutzes konzipieren, während von einer anderen, ebenso kritischen Seite Grenzen als Instrument der Aus- und Abgrenzung verstanden werden. Ein universalistischer Ansatz, der sich zum Teil auf die sozialwissenschaftliche Tradition in der Grenzforschung, wie sie weiter oben dargestellt wurde, und auf ethische Grundlagen humanitärer Solidarität beruft (vgl. Paasi 2019), stellt sich gegen die Konsequenzen einer nationalen Grenzpolitik. Besonders Mobilität und Netzwerke sind die zentralen Kategorien einer weit verbreiteten sozialwissenschaftlichen Sichtweise, die auch die Öffentlichkeit erreicht hat (vgl. Nail 2016). Aus dieser Perspektive werden die „space of flows“ (Castells 1996) von Gütern und Informationen, transnationale Räume und Prozesse und die neue Qualität von Grenzüberschreitungen betont. Vor allem werden die weltweiten Migrationsbewegungen hervorgehoben, die eine Reaktion auf die schwierigen Lebensbedingungen in vielen Herkunftsländern sind.

Verschiedene kritische Forschungen zu Migration und Grenzregimen (z.B. Tsianos/Karakayali 2008; Tsianos/Hess 2010) betonen nicht nur, dass diese Überschreitungen eine nicht zu verhindernde Realität sind - dem Umstand geschuldet, dass Grenzen niemals geschlossen werden können und allenfalls reguliert werden. Sie sehen außerdem in diesen Entwicklungen eine Produktivität der Grenzgänger*innen und Grenzüberschreitungen für ökonomische und kulturelle Prozesse und kritisieren ,normativistische ${ }^{`}$ Vorstellungen. Hier werden die Praktiken des doing border mit Untersuchungen zu Regierungspraktiken und den Verhaltensweisen und Strategien 
der Geflüchteten methodisch in den Vordergrund gestellt. In diesem Perspektivwechsel sind auch moralische Vorstellungen virulent, wenn die Grenzpolitik gegenüber Migrant*innen aus Sicht von Menschenrechten bzw. einer grundlegenden Humanität als undemokratisch bewertet wird (vgl. Paasi 2019). So verstanden wären Grenzen nicht legitimierte politische Instrumente, auch weil sich die Bedingungen des Konzepts Grenze verändert haben, wie Étienne Balibar (2003) analysiert. Der Vorstellung von Grenze liegt nach dieser Einschätzung nicht mehr eine grundlegende territorial begründete Trennung von außen und innen zugrunde, weil migrantische Realitäten die Vorstellungen von nationalen Zugehörigkeiten und Territorien infrage stellen. ${ }^{10}$

In diesem Zusammenhang ist auch die politisch-rechtliche Veränderung des Grenzregimeverständnisses Gegenstand diverser Einschätzungen, die die Legitimation von neuen staatlichen Grenzpraktiken diskutieren. Es werden aus einer politischen und juristischen Perspektive konzeptuelle Veränderungen von zumindest europäischen Grenzregimen in die Analyse einbezogen, wenn Grenzen zunehmend privatisiert, internalisiert und externalisiert werden. Verena Risse (2018) folgend, kann man zusammenfassen, dass Grenzkontrollen immer weiter privatisiert werden; deutlich zu beobachten ist das etwa bei Fluggesellschaften. Die Externalisierung zeigt sich darin, dass Grenzkontrollen schon vor dem eigentlichen Hoheitsgebiet durchgeführt werden. Als Internalisierung kann gelten, wenn der Aufenthalt und die Meldepflicht im Inland kontrolliert und nationale Grenzen ausgeweitet werden.

Die Veränderungen sind durch die herkömmlichen rechtlichen Strategien wenig legitimiert. Diese gesellschaftspolitischen Änderungen weisen auf die Probleme von Legitimitätsdiskursen hin, die entstehen, wenn ein Zustand behauptet wird, der die Notwendigkeit nationaler Grenzschließungen oder antihumanitärer Maßnahmen impliziert, sodass die Maßnahmen und Schließungen nicht eingehender gerechtfertigt werden müssen, weil der Notstand die Praxis der Grenzziehung quasi als naturwüchsig erscheinen lässt (vgl. Brown 2018). Eine politischphilosophische Debatte (vgl. Abizadeh 2013) wirft dementsprechend auch ethische Fragen zur Rechtfertigungsbedürftigkeit von Grenzmaßnahmen auf, weil die Beschränkungen der Bewegungsfreiheit etwa Grundrechte und Menschenrechte herausfordern. Grenzen können nicht der Forderung nach demokratischer Legitimierung entzogen werden, so die Kritik.

Die beschriebenen konträren normativen Vorstellungen des nationalen souveränen Grenzkonzepts auf der einen und des universalistischen Ansatzes auf der anderen Seite sowie empirische Studien zu Grenzpraktiken zeigen, dass die Grenzpolitik vieler Staaten und Staatenbündnisse der Öffentlichkeit gegenüber gerechtfertigt werden muss, vor allem, wenn Menschen bei der Grenzüberquerung sterben oder in Auffanglagern stranden (vgl. Vobruba 2006; Mezzadra 2018). Das Selbstverständnis der Staaten und Staatenbündnisse wird durch transnationale Migration herausgefordert. Die nationale Grenze wird zum symbolischen Gegenstand für Bewertungen von Migration, Staaten und Sicherheitsdispositiven und deren Legitimationen. Besonders gesundheitspolitische Vorgänge, die mit den Schutz- und Sicherheitsmaßnahmen verknüpft werden, sind Teil einer Eigendynamik von nationalen Grenzen, die in dem Feld der Medizin neben den exkludierenden Effekten auch inkludierende Abstufungen zulassen und neue Grenzziehungen zwischen verschiedenen Grenzgänger*innen ermöglichen. Wenn die Herkunft etwa bei der Versorgung von schwerkranken Geflüchteten oder Migrant*innen relevant wird, weil ein Pass Leistungen garantiert, die Patient*innen ohne Pass nicht bekommen,

10 Eine ähnliche Perspektive nimmt Giorgio Agamben (2001) ein, der vor allem die kaum legitimierbare Rechtsform des Ausnahmezustands als Rechtfertigung für die Flüchtlingslager sieht. 
selektiert das Gesundheitssystem nach politisch-nationalen Kriterien auf Grundlage nationaler Grenzen (vgl. Banse 2018). Wenn, so kann man zusammenfassen, universelle Normen verletzt werden, die im Zusammenhang mit Migration stehen, wie Bewegungsfreiheit, der Schutz vor Verfolgung oder das Gebot, kranken Menschen die nötige medizinische Versorgung zu ermöglichen, braucht es „scharfe legitimatorische Waffen“ (Pfau 2008, S. 40).

\subsection{Legitimationsprobleme in Grenzregionen}

Auch in europäischen Grenzregionen als Lebensmittelpunkt geht es in öffentlichen regionalen Kontroversen um die schwindende normative Legitimität von nationalen Grenzen, deren Bedeutung wie im Migrationsdiskurs im ,moralischen“ Ton diskutiert werden kann, etwa indem auch hier der Schutz der Bevölkerung gegen eine grenzüberschreitende Bedrohung geltend gemacht wird. Themen in diesem Zusammenhang sind etwa in europäischen Grenzregionen Schleuserkriminalität (Pfau 2008), Schmuggel (Irek 1998; Wagner 2011), Prostitution (Pates/Schmidt 2009) und auch hier (Pendel-)Migration (Dall Schmidt 2006). ${ }^{11}$ Anders als im Konflikt um Effekte von Grenzregimen geht es in den Grenzregionen in der Regel nicht nur um Ausnahmezustände in Krisenzeiten, sondern auch um alltägliche Vorstellungen und Handlungen, die mit Grenzen zusammenhängen. Normative Legitimationskonflikte spielen sich auf verschiedenen Ebenen ab, die im Hinblick auf die Funktion der Grenze empirisch differenziert werden können, wenn die Vielzahl der Akteure und ihre Interessen einbezogen werden.

Zunächst stehen sich an der trennenden nationalen Grenze Staaten gegenüber, Grenzregionbewohner*innen erleben die Grenzlinie als etwas, das erst geschaffen werden musste. Als Symbole nationaler Politik haben Grenzen praktische Konsequenzen, weil zunächst Unterschiede geschaffen und sozial etabliert werden (Baud/Schendel 1997). Häufig wurde einmal um die Rechtmäßigkeit der Linie, die sie trennt, gestritten, zuweilen gekämpft. Die meisten der die Staaten trennenden Grenzen haben eine konfliktreiche Geschichte, die von kontroversen Legitimationserzählungen begleitet wird. Gerade Grenzziehungen, die aus der Zeit des Nationalsozialismus stammen, zeigen die nachhaltige und ambivalente Wirkung von Grenzdiskussionen, die von Heimat und Vertreibung handeln (vgl. Brumlik 2005). Wenn es um die historische Begründung der Staatsgrenze und ihres Verlaufs geht, sind die häufig aus Kriegen oder dem Zerfall von Imperien entstandenen Grenzziehungen Gegenstand schwer lösbarer Auseinandersetzungen staatlicher Akteure um nicht verhandelbare Grenzen (vgl. Bös/Zimmer 2006; Bach 2010). Georg Simmels (1908/1992) in der Zeit größter nationaler Konflikte am Anfang des 20. Jahrhunderts verfasster abstrakte Gedanke, dass die Grenze nicht das Ende, sondern den Anfang eines Streits darstellt, findet seine empirische Konkretisierung in vielen europäischen Grenzregionen. Die staatlichen Akteure haben manchmal deutlich widersprechende Einschätzungen über den Grenzverlauf, der begründet werden muss. International politisch anerkannte Legitimationen für Grenzveränderungen (vgl. Bös/Zimmer 2006) beziehen sich meist auf historische Ereignisse, die im Zusammenhang mit der Selbstbestimmung der Nation stehen (vgl. Bielefeld 2003). Je nach historischem Kontext der Grenzziehung unterscheiden sich diese Legitimierungen jedoch, die Konflikte verweisen auf ganz verschiedene Auseinandersetzungen und ,Kämpfe', die für die Bevölkerung eine symbolische Kraft auch ,nach innen` entfalten können, besonders, wenn sie an die nationale Erzählung anknüpfen. In den Grenzregionen

11 Vgl. auch allgemein zu dem Thema Grenzverletzungen an Staatsgrenzen Eigmüller (2008). 
innerhalb der Europäischen Union finden sich lange etablierte nationale Differenzen, die sich in den Meinungen über den Nachbarn widerspiegeln (vgl. Rüdiger 2004; Roose 2010). Wenn ,billige‘ Konkurrenz (wie etwa an der polnisch-deutschen Grenze im Konflikt zwischen Bäckern) gefürchtet und die kulturelle Differenz zu Mehrheiten von Minderheiten in der dänischdeutschen Grenzregion betont wird, bleiben bipolare Sichtweisen eine wichtige Folie, auf der Abgrenzungen legitimiert werden. Gleichwohl ist diese normative Legitimität der nationalen Grenze nicht selbstverständlich, vielmehr ein widersprüchlicher Prozess, der im regionalen, historischen und politischen Kontext gesehen werden muss.

Historisch mussten Grenzen in Europa in den entstehenden Grenzregionen von Nationalstaaten erst etabliert werden. Legitime Ansprüche auf Eigentum, wie sie in mittelalterlichen Zeiten traditionell vorherrschten, wurden nun zentral geregelt (vgl. Landwehr 2006). Es gibt viele Grenzregionen auf der Welt und auch in Europa, deren Bewohner*innen der nationalen Zentrumspolitik (wie auch heute der Europapolitik) kritisch gegenüberstanden und -stehen (vgl. Sahlins 1989; Banse 2013, S. 266-278). Die Legitimation nach innen, die der Nationalstaat politisch fördern musste, basierte auf nationalen Bezügen, mit denen sprachlich-kulturelle Unterschiede auf dem Hoheitsgebiet etabliert werden sollten. Grenzen wurden in der europäischen Geschichte der Nationalstaaten immer wieder militarisiert. Ehre und Würde, wie Lucien Febvre (1988) schreibt, machten die Grenze zwischen Nationen zu „moralischen Grenzen“ (ebd., S. 33, Herv. CB); im Kriegsfall sollten sie von nun an mit dem eigenen Leben geschützt werden. Gerade Bedrohungsszenarien, die etwa auch mit vorherigen Grenzverschiebungen und militärischen Grenzüberschreitungen, also mit der Ungewissheit zusammenhingen, die in Europa bei Grenzziehungen herrschte, mobilisierten Akteure zu politisch-ethnischen Gemeinschaften. Die historische Legitimation von nationalen Grenzen hat heute noch immer eine mobilisierende Wirkung, wenngleich oder weil der Rahmen ein ganz anderer ist, wenn im Zusammenhang mit der Europäischen Integration die einstigen Gegensätze überwunden werden sollen.

Dabei sollte deutlich werden, dass regionale Legitimierungen nationaler Grenzen sich nach dem Kontext der historischen Situation unterscheiden. Wenn etwa die nationalen Grenzen von Deutschland zu Frankreich, Dänemark und Polen verglichen werden (siehe Banse 2013), so hat jede der noch heute aktuellen Grenzen eine spezifische Entstehungsgeschichte, sodass die Grenzen deshalb historisch auf jeweils andere Weise legitimiert oder delegitimiert werden. Die Grenze zwischen Frankreich und Deutschland hat eine lange Tradition an unklaren Grenzbestimmungen; besonders das Elsass mit seiner wechselvollen Geschichte zeigt, dass Staatsgrenzen wandern und Zugehörigkeiten variieren - wie die wechselvollen Familiengeschichten der Elsässer in der Grenzregion verdeutlichen (vgl. ebd., S. 108f.). Die dänisch-deutsche Grenze wiederum entstand nach einer Volksabstimmung 1920 und konnte nur dadurch relativ konfliktfrei vollzogen werden, indem der deutschen und dänischen Minderheit auf der jeweils anderen Seite der trennenden Grenze ausreichend Rechte zugesprochen wurden. Schließlich ist die polnisch-deutsche Grenze Resultat des Zweiten Weltkriegs und Ausdruck der Willkür großräumiger politischer Entscheidungen, die ganze Bevölkerungen über Grenzen wandern ließen und Städte ,wie mit dem Rasiermesser' scharf trennten. Abgrenzungen sind das Resultat einer kaum geleisteten Bewältigung von Kriegserfahrungen (vgl. ebd., S. 224ff.). Ob das Versterben der Kriegsgenerationen europäische und grenzüberschreitende Politik legitimieren, wird die Forschung beschäftigen. 
Durch die Europäische Integration wurden diese Grenzen in der EU geöffnet. Konzepte zur grenzüberschreitenden Zusammenarbeit und zum transnationalen Regionalismus sollen die nationale Konfrontation in der Grenzregion relativieren und nun Grenzüberschreitungen legitimieren (vgl. Ulrich/Scott in diesem Band). Wie virulent nationale Grenzen weiterhin sind, zeigt sich in einem bemerkenswerten Paradox der Europäischen Integration, das an die eben genannten Konflikte anschließt und ein Licht auf Legitimitätsfragen wirft. Vielen Grenzregionen ist gemeinsam, dass die Vielzahl heutiger offizieller Legitimationskämpfe in europäischen Grenzregionen sich darauf beziehen, dass die Anerkennung der nationalen Grenze eine Voraussetzung für erlaubte Grenzüberschreitungen ist; um auf grenzüberschreitende Kooperation zu hoffen, muss die trennende Grenze politisch von der anderen Seite legitimiert sein. So hat Dänemark die Anerkennung der Grenze und des Status der Minderheit in Deutschland im Zusammenhang mit dem Selbstbestimmungsrecht der Völker als Voraussetzung für weiteren Austausch gesehen (vgl. Kühl 2004); auch die polnische Seite hat Deutschland zu einem entsprechenden Umgang mit der aus dem Zweiten Weltkrieg entstandenen Grenze aufgefordert und wollte die nationale Grenze politisch anerkannt wissen (vgl. Banse 2013, S. 203ff.). Vor allem vor dem Hintergrund gewaltsamer Grenzüberschreitungen durch das nationalsozialistische Deutschland wird eine vorsichtige Grenzpolitik, auch im grenzüberschreitenden Kontext der Europäisierung, von den einst angegriffenen Staaten politisch legitimiert.

Die Betrachtung von multidimensionalen und eigendynamischen Legitimationsprozessen in Grenzregionen stellt sich empirisch weitaus komplexer dar als die Betrachtung der reinen staatlichen Steuerungsperspektive, wenn nichtstaatliche Akteure, die in den Grenzregionen leben, einbezogen werden. Sie verfolgen regionale oder eigene Interessen, die denen der Staaten widersprechen können. In Grenzregionen rücken Grenzüberschreitungen, subversive Ökonomien (vgl. Eigmüller 2008) und alltägliche Aushandlungen von Vorteilen für die Bewohner*innen in den Vordergrund, der einen anderen Blick auf Legitimitätsprobleme gestattet: Grenzüberschreitungen können regional legitimiert sein, auch wenn sie es in einem größeren politischen Rahmen nicht sind, etwa, weil sie illegal sind. Dieses Phänomen betrifft etwa die beiderseitige Anerkennung von Prostitution in der polnisch-deutschen Grenzregion, weil zum Beispiel der finanzielle Gewinn bei Wohlstandsunterschieden bei den ,Anbietern' oder weil die Nutzung von Preis- und Rechtsunterschieden bei den ,Kunden` als legitim angesehen wird. ,Tanktouristen` wie ,Schnäppchenjäger‘ sind ,legitime‘ Bestandteile von Grenzökonomien.

Auch hier ist die nationale Grenze Voraussetzung für ihre Überschreitung. Der Unterschied, der durch die Grenzziehung geschaffen wurde, ökonomisch, politisch, rechtlich und zuweilen auch sozial und kulturell, kann für den regionalen Austausch der Grenzregionsbewohner*innen produktiv sein, wenn er etwas ermöglicht, was auf der eigenen Seite nicht möglich ist. Zugleich können sich sogar über diese Ökonomien gemeinsame grenzüberschreitende Interessen beider getrennten Grenzregionen bilden, die auch gegen jeweilige Zentrumsinteressen artikuliert werden (vgl. Sahlins 1989). Dementsprechend wird eine Grenzpolitik in der Region auch andere (zusätzliche) legitimierende Begründungen für die Grenze formulieren, als der rein nationalstaatliche ,abgrenzende‘ oder der europäische ,grenzauflösende‘ Blick es tun.

Die Erforschung europäischer Grenzregionen zeigt, dass die Legitimationsdiskurse auf der einen Seite stark von den Entstehungsbedingungen der politischen Grenzen abhängen und bei Kontroversen national kodiert werden. Diese Bedingungen spielen bei der Delegitimierung von europäischer Politik in den Grenzregionen eine Rolle, wenn symbolisch die nationale Grenze als Schutzzone vorgestellt wird und verschiedene Abgrenzungen vorgenommen werden. 
Auf der anderen Seite herrschen jedoch spezifische Bedingungen vor Ort vor, die den multidimensionalen Charakter von nationalen Grenzen verdeutlichen können, der vor allem heute relevant und vielleicht immer relevanter wird. Die Grenzlegitimität ist mit einem Netzwerk unterschiedlicher und komplex vernetzter Legitimationsprozesse verbunden, die noch weitgehend Forschungsdesiderat sind. So stehen Grenzen im lokalen Fokus, weil die Anrainer*innen durch eigene Entscheidungen den Grenzübertritt für legitim befinden, der ihren Interessen entspricht. Regional legitim kann der Bezug zu ökonomischen Standortbestimmungen sein, die den Ort auf- oder abwerten, während europäische Legitimationen hieran zwar anschließen können, jedoch auch im Konflikt mit den regionalen Prozessen stehen, wenn die Europäische Union symbolisch oder real noch wenig in der Region etabliert ist. Es kann durchaus sein, dass die Europäische Union als bürokratischer Apparat regional abgelehnt wird, wie es etwa in Dänemark der Fall ist (vgl. Kühl 2004). Zugleich sind Finanzmittel der Europäischen Union begehrte Hilfen für die Region und legitimieren politische Ideen der Zusammenarbeit. Schließlich sind Grenzregionen Akteure bei globalen kapitalistischen Marktprozessen, die Standorte in einen weltweiten Konkurrenzkampf bringen. Dass diese ökonomischen und politischen Auseinandersetzungen wiederum in nationale Deutungen, Abwehrkämpfe und Abgrenzungen eingebettet werden, kann unter den erläuterten Umständen als eine Form der Simplifizierung gesehen werden, die sich leichter ,moralisch`verwenden lässt und Ressentiments rechtfertigen hilft.

\section{Fazit und Ausblick}

Eine vor allem sozialwissenschaftliche und grenzsoziologische Perspektive auf die Frage nach der normativen wie empirischen Legitimität von (nationalen) Grenzen in der Gesellschaft heute sollte bedenken, welche der Erkenntnisse für weitere gesellschaftstheoretische Beobachtungen wichtig sein könnten. Diese Perspektive sollte über die deskriptive Darstellung der Legitimationsprobleme von nationalen Grenzen hinausgehen. Wichtig ist die Frage: Warum werden Grenzen von Nationalstaaten überhaupt legitimiert? Oder anders gefragt: Warum haben gerade nationale Grenzen Legitimierungsprobleme? ${ }^{12}$

Nach dem sozial- und grenzwissenschaftlichen Forschungsstand sind nationale Grenzen heute ohne Frage besonders legitimierungsbedürftig, wenn man einen Maßstab anlegt, der sich an den öffentlich-politischen und den wissenschaftlichen Diskussionen orientiert. Beide hier diskutierten Themenfelder, Migration wie Grenzregion, offenbaren den Legitimierungsdruck, unter dem Grenzöffnungen wie -schließungen stehen. Offensichtlich wird ihre Legitimität zunehmend problematisch, weil die mehrdimensionale und widersprüchliche Realität der nationalen Grenze in den Blick kommt und keine ,einfache' Legitimation möglich ist, die etwa nur auf der nationalen Rechtfertigung basiert. Die nationale Grenze wird zum Gegenstand von Aushandlungen (vgl. Bach 2010), wenn verschiedene Perspektiven einbezogen und gegenübergestellt werden. Hier müssen Forschungen anschließen und die Grenzdiskurse und die Diversität an Legitimierungen fassen.

Systematisch sollte im Rückblick der Beispiele in diesem Beitrag festgehalten werden: Grenzen schlichten nicht nur Konflikte, sondern sie polarisieren auch, weil sie Unterscheidungen und Gegensätze, die oft auch lebensbedrohliche Konsequenzen haben können, etablieren wie kaum ein anderes politisch-soziales Instrument. So gesehen symbolisieren Grenzen einerseits

12 Diese Frage kann hier natürlich nicht beantwortet werden; es wäre dazu nötig, auch andere Institutionen der heutigen Gesellschaft einer Legitimitätsprüfung zu unterziehen. 
normative Positionen, deren kontroverse Rechtfertigungsdynamiken andererseits anzeigen, dass sie immer weniger selbstverständlich sind. Hier sollten Rechtfertigungsformen in den Blick genommen werden, um die Semantik des Grenzbegriffs stärker auf seine widersprüchliche Bedeutung hin zu analysieren. So kann etwa eine Mauer als Grenze den souveränen Nationalstaat symbolisieren, während zugleich differenzierte grenzüberschreitende Prozesse an derselben Grenze stattfinden, die seine Souveränität unterlaufen. Nationale Grenzen werden in einer praxeologischen Weise sozial dadurch legitimiert, dass Akteure auf sie bezogen handeln, sie überschreiten, weil sie einen Unterschied markieren, den man ,pragmatisch' nutzen kann. Die Grenze ist dann in einer unmittelbaren Weise legitim, weil ihre Überschreitung Chancen generiert. ${ }^{13}$ Gerade Forschungen in binneneuropäischen Grenzregionen zeigen, dass trennende Grenzen auch durchaus eine Funktion im psychischen Haushalt vieler Menschen haben und dementsprechend auch, gewünscht‘ sind.

Grenzen sind moralisch. Sie sind also nicht nur Ausdruck unterschiedlicher Bedürfnisse und auch Sichtweisen, die an der Grenze aufeinanderprallen, sondern Grenzen selbst sind eine Form der moralischen Vorstellung, in der schließlich davon ausgegangen wird, dass etwas zu einem gehört oder nicht gehört, dass etwas nicht weitergehen darf oder überschritten werden soll und wo Eindeutigkeit oder Komplexität herrschen. Dass und wie diese Moral und Bewertung zunehmend (und nicht nur in Grenzregionen) ausgehandelt werden, muss Grundlage weiterer Grenzforschungen sein, die nach der sozialen Herstellung der Legitimität von Grenzen fragen.

Die Forschung muss dabei reflektieren, in welcher Perspektive sie die Grenze betrachtet, etwa aus einer partizipierenden und zielorientierten und damit unter Umständen Moral verstärkenden Perspektive oder aus einer Perspektive, die die moralische Dimension von Grenzen reflektiert, indem sie selbst eine Perspektivenvielfalt berücksichtigt. An Grenzen treffen unterschiedliche Legitimitätsvorstellungen aufeinander: Sie sind der Ausdruck und Auslöser von moralisch konnotierten Konflikten, die gesellschaftliche Sprengkraft haben - je mehr das Normative und die Legitimität selbst an ihre Grenzen kommen.

\section{Weiterführende Literatur}

Abizadeh, Arash (2013): Geschlossene Grenzen, Menschenrechte und demokratische Legitimation. In: polylog. Zeitschrift für interkulturelles Philosophieren 30, S. 5-23.

Brown, Wendy (2018): Mauern. Die neue Abschottung und der Niedergang der Souveränität. Berlin: Suhrkamp.

Dammayr, Maria/Graß, Doris/Rothmüller, Barbara (2015): Legitimität und Legitimierung in der sozialwissenschaftlichen Debatte: eine Einführung in Theorien der Rechtfertigung und Kritik von Herrschaft. In: Dies. (Hrsg.): Legitimität. Gesellschaftliche, politische und wissenschaftliche Bruchlinien der Rechtfertigung. Bielefeld: transcript, S. 7-24.

Paasi, Anssi/Prokkola, Eeva-Kaisa/Saarinen, Jarkko/Zimmerbauer, Kaj (Hrsg.) (2019): Borderless Worlds for Whom? Ethics, Moralities and Mobilities. London: Routledge.

\section{Literaturverzeichnis}

Abizadeh, Arash (2013): Geschlossene Grenzen, Menschenrechte und demokratische Legitimation. In: polylog. Zeitschrift für interkulturelles Philosophieren 30, S. 5-23.

Agamben, Giorgio (2001): Mittel ohne Zweck. Noten zur Politik. Freiburg/Berlin: diaphanes.

13 In diesem Zusammenhang muss betont werden, dass Grenzüberschreitungen auch dadurch legitimiert werden, dass ihre Überschreitung als rettende Maßnahme gelten kann, wie etwa bei Fluchthandlungen aus dem nationalsozialistischen Deutschland oder aus der DDR. Hier wird deutlich, dass sich der Kontext Grenzhandlungen ganz unterschiedlich bewerten lässt. 
AGEG (Hrsg.) (2008): Zusammenarbeit Europäischer Grenzregionen. Bilanz und Perspektiven. Baden-Baden: Nomos.

Bach, Maurizio (2010): Die Konstitution von Räumen und Grenzbildung in Europa. Von verhandlungsresistenten zu verhandlungsabhängigen Grenzen. In: Eigmüller, Monika/Mau, Steffen (Hrsg.): Gesellschaftstheorie und Europapolitik. Sozialwissenschaftliche Ansätze zur Europaforschung. Wiesbaden: VS Verlag für Sozialwissenschaften, S. 153-178.

Balibar, Etienne (2003): Sind wir Bürger Europas? Politische Integration, soziale Ausgrenzung und die Zukunft des Nationalen. Hamburg: Hamburger Edition.

Banse, Christian (2013): Nationale Grenzerfahrungen und grenzüberschreitende Prozesse: Eine soziologische Untersuchung an ausgewählten Grenzregionen. Frankfurt/M.: Peter Lang.

Banse, Christian (2018): Komplexe Grenzziehungen und ungewisse Grenzdynamiken. Zur Palliativversorgung von Menschen mit Migrationshintergrund und Geflüchteten. In: Berliner Debatte Initial 29, H. 1, S. 84-94.

Banse, Christian/Müller, Doreen/Stobbe, Holk (2007): Tödliche Vision Europa. Die grenz-, migrationsund asylpolitischen Komponenten der Europäischen Nachbarschaftspolitik. In: Fischer, Robert/Karrass, Anne/Kröger, Sandra (Hrsg.): Die europäische Kommission und die Zukunft der EU: Ideenfabrik zwischen europäischem Auftrag und nationalen Interessen. Opladen/Farmington Hills: Verlag Barbara Budrich, S. 179-203.

Baud, Michiel/Schendel, Willem van (1997): Toward a Comparative History of Borderlands. In: Journal of World History 8, H. 2, S. 211-242.

Beck, Ulrich (1999): Was ist Globalisierung? Irrtümer des Globalismus - Antworten auf Globalisierung. Frankfurt/M.: Suhrkamp.

Bielefeld, Ulrich (2003): Nation und Gesellschaft. Selbstthematisierungen in Deutschland und Frankreich. Hamburg: Hamburger Edition.

Bös, Mathias/Zimmer, Kerstin (2006): Wenn Grenzen wandern. Zur Dynamik von Grenzverschiebungen im Osten Europas. In: Eigmüller, Monika/Vobruba, Georg (Hrsg.): Grenzsoziologie. Die politische Strukturierung des Raumes. Wiesbaden: VS Verlag für Sozialwissenschaften, S. 157-184.

Boltanski, Luc/Thévenot, Laurent (2007): Über die Rechtfertigung. Eine Soziologie der kritischen Urteilskraft. Hamburg: Hamburger Edition.

Bourdieu, Pierre (2014): Über den Staat. Vorlesungen am Collège de France 1989-1992. Berlin: Suhrkamp.

Brown, Wendy (2018): Mauern. Die neue Abschottung und der Niedergang der Souveränität. Berlin: Suhrkamp.

Brumlik, Micha (2005): Wer Sturm sät. Die Vertreibung der Deutschen. Berlin: Aufbau Verlag.

Castells, Manuel (1996): The Rise of the Network Society. Cambridge/Oxford: Blackwell.

Dall Schmidt, Torben (2006): Wer ist der Grenzpendler an der deutsch-dänischen Grenze? Grenzpendeln von Deutschland nach Sønderjylland 1998-2003. Aabenraa: Institut for Grænseregionsforskning og forfatteren.

Dammayr, Maria/Graß, Doris/Rothmüller, Barbara (2015): Legitimität und Legitimierung in der sozialwissenschaftlichen Debatte: eine Einführung in Theorien der Rechtfertigung und Kritik von Herrschaft. In: Dies. (Hrsg.): Legitimität. Gesellschaftliche, politische und wissenschaftliche Bruchlinien der Rechtfertigung. Bielefeld: transcript, S. 7-24.

Dux, Günter (2010): Demokratietheorie und Europäische Integration. Zur Dekonstruktion des Demos. In: Eigmüller, Monika/Mau, Steffen (Hrsg.): Gesellschaftstheorie und Europapolitik. Sozialwissenschaftliche Ansätze zur Europaforschung. Wiesbaden: VS Verlag für Sozialwissenschaften, S. 49-79.

Eigmüller, Monika (2008): Subversionen an Staatsgrenzen - eine Einleitung. In: Comparativ. Zeitschrift für Globalgeschichte und vergleichende Gesellschaftsforschung 18, S. 13-22.

Falk, Francesca (2011): Eine gestische Geschichte der Grenze. Wie der Liberalismus an der Grenze an seine Grenzen kommt. München: Wilhelm Fink.

Fassin, Didier (2020): Introduction: Connecting Borders and Boundaries. In: Ders. (Hrsg.): Deepening Divides. How Physical Borders and Social Boundaries Delineate our World. London: Pluto, S. 1-18.

Febvre, Lucien (1988): Das Gewissen des Historikers. Berlin: Wagenbach.

Flügel-Martinsen, Oliver/Kramer, Kirsten/Vasilache, Andreas (2018): Grenzprozesse in der Weltgesellschaft. Theoretische Anmerkungen zum Begriff der Grenze. In: Berliner Debatte Initial 29, H. 1, S. $12-$ 25.

Forst, Rainer (2015): Normativität und Macht. Zur Analyse sozialer Rechtfertigungsordnungen. Berlin: Suhrkamp.

Gerst, Dominik/Klessmann, Maria/Krämer, Hannes/Sienknecht, Mitja/Ulrich, Peter (2018): Komplexe Grenzen. Aktuelle Perspektiven der Grenzforschung. In: Berliner Debatte Initial 29, H. 1, S. 3-11.

Graziano, Manlio (2018): What is a Border? Stanford: Stanford University Press. 
Habermas, Jürgen (1971): Legitimationsprobleme im Spätkapitalismus. Frankfurt/M.: Suhrkamp.

Heyman, Josiah M./Symons, John (2015): Borders. In: Fassin, Didier (Hrsg.): A Companion to Moral Anthropology. Hoboken: Wiley-Blackwell, S. 540-557.

Irek, Malgorzata (1998): Der Schmugglerzug Warschau-Berlin-Warschau. Berlin: Das Arabische Buch.

Kaufmann, Stefan/Bröckling, Ulrich/Horn, Eva (2002): Einleitung. In: Dies. (Hrsg.): Grenzverletzer. Von Schmugglern, Spionen und andern subversiven Gestalten. Berlin: Kadmos, S. 7-22.

Koschorke, Albrecht (2017): Wahrheit und Erfindung. Grundzüge einer Allgemeinen Erzähltheorie. 4. Aufl., Frankfurt/M.: S. Fischer.

Kühl, Jørgen (2004): Dänisch-Deutsche Grenzregion: Erfahrungen zwischen Deutsch und Dänisch. In: Banse, Christian/Stobbe, Holk (Hrsg.): Nationale Grenzen in Europa. Frankfurt/M.: Peter Lang, S. 6796.

Landwehr, Achim (2006): Der Raum als „genähte“ Einheit: Venezianische Grenzen im 18. Jahrhundert. In: Behrisch, Lars (Hrsg.): Vermessen, Zählen, Berechnen. Die politische Ordnung des Raums im 18. Jahrhundert. Frankfurt/M./New York, S. 45-64.

Luhmann, Niklas (1982): Territorial Borders as System Boundaries. In: Strassoldo, Raimondo/Zotti, Giovanni Delli (Hrsg.): Cooperation and Conflict in Border Areas. Milano: Angeli, S. 237-244.

Luhmann, Niklas (2000): Die Politik der Gesellschaft, Frankfurt/M.: Suhrkamp.

Mann, Michael (1997): Has globalization ended the rise of the nation-state? In: Review of International Political Economy 4, H. 3, S. 472-496.

Mau, Steffen (2006): Die Politik der Grenze. Grenzziehung und politische Systembildung in der Europäischen Union. In: Berliner Journal für Soziologie 16, H. 1, S. 115-132.

Mezzadra, Sandro (2018): Sealing Borders? Rethinking Border Studies in Hard Times. Working Paper Series B/ORDERS IN MOTION Nr. 3, Frankfurt/Oder: Viadrina. DOI:10.11584/B-ORDERS. 3.

Nail, Thomas (2016): Theory of the border. Oxford/New York: Oxford University Press.

Newman, David/Paasi, Anssi (1998): Fences and Neighbours in the Postmodern World: Boundary, Narratives in Political Geography. In: Progress in Human Geography 22, H. 2, S. 186-207.

Ohmae, Kenichi (1990): The Borderless World: Power and Strategy in the Interlinked Economy. New York: Harper Business.

Paasi, Anssi (2019): Borderless Worlds and Beyond: Challenging the State-Centric Cartographies. In: Ders./Prokkola, Eeva-Kaisa/Saarinen, Jarkko/Zimmerbauer, Kaj (Hrsg.): Borderless Worlds for Whom? Ethics, Moralities and Mobilities. London: Routledge, S. 21-36.

Pates, Rebecca/Schmidt, Daniel. (2009): Die Verwaltung der Prostitution. Eine vergleichende Studie am Beispiel deutscher, polnischer und tschechischer Kommunen. Bielefeld: transcript.

Pfau, Jonas (2008): Prekäre Migration und Ausschluss: Die gesellschaftliche Perzeption von illegaler Migration, Fluchthilfe und Menschenschmuggel in und nach Mitteleuropa. In: Comparativ. Zeitschrift für Globalgeschichte und vergleichende Gesellschaftsforschung 18, S. 23-41.

Risse, Verena (2018): Die Diversifikation von Staatsgrenzen - Anlass zu einer konzeptionellen Neubestimmung? In: Berliner Debatte Initial 29, H. 1, S. 62-72.

Roose, Jochen (2010): Vergesellschaftung an Europas Binnengrenzen. Wiesbaden: VS Verlag für Sozialwissenschaften.

Rüdiger, Jan (2004): Vom Nutzen des Vergessens. Schleswig-Holsteins Landesmittelalter. In: Lundt, Bea (Hrsg.): Nordlichter. Geschichtsbewußtsein und Geschichtsmythen nördlich der Elbe. Köln/Weimar/ Wien: Böhlau, S. 87-135.

Sahlins, Peter (1989): Boundaries. The Making of France and Spain in the Pyrenees. Berkeley: University of California Press.

Schimank, Uwe (2005): Weltgesellschaft und Nationalgesellschaften: Funktionen von Staatsgrenzen. In: Zeitschrift für Soziologie, Sonderheft Weltgesellschaft, S. 394-415.

Schroer, Markus (2006), Räume, Orte, Grenzen. Auf dem Weg zu einer Soziologie des Raums. Frankfurt/M.: Suhrkamp.

Simmel, Georg (1908/1992): Soziologie. Untersuchungen über die Formen der Vergesellschaftung. Frankfurt/M.: Suhrkamp.

Schultz, Hans-Dietrich (1993): Deutschlands „natürliche“ Grenzen. In: Demandt, Alexander (Hrsg.): Deutschlands Grenzen in der Geschichte. München: Beck, S. 32-94.

Tsianos, Vassilis/Karakayali, Serhat (2008): Die Regierung der Migration in Europa Jenseits von Inklusion und Exklusion. In: Soziale Systeme 14, H. 2, S. 329-348.

Tsianos, Vassilis/Hess, Sabine (2010): Ethnographische Grenzregimeanalysen. Eine Methodologie der Autonomie der Migration. In: Hess, Sabine/Kasparek, Bernd (Hrsg.): Grenzregime. Diskurse, Praktiken, Institutionen in Europa. Berlin: Assoziation A, S. 243-264.

Vasilache, Andreas (2007): Der Staat und seine Grenzen. Zur Logik politischer Ordnung. Frankfurt/M./New York: Campus. 
Velde, Martin van der/Houtum, Henk van (Hrsg.) (2000): Borders, Regions, and People, London: Pion. Vobruba, Georg (2006): Grenzsoziologie als Beobachtung zweiter Ordnung. In: Eigmüller, Monika/ders.: Grenzsoziologie. Die politische Strukturierung des Raumes. Wiesbaden: VS Verlag für Sozialwissenschaften, S. 217-225.

Wagner, Mathias (2011): Die Schmugglergesellschaft. Informelle Ökonomien an der Ostgrenze der Europäischen Union. Bielefeld: transcript.

Weber, Max (1921/1980): Wirtschaft und Gesellschaft. Grundriss der verstehenden Soziologie. 5. rev. Aufl. Tübingen: Mohr.

Yarbrough, Beth V./Yarbrough, Robert M. (2003): The Contractual Role of Boundaries: Law and Economic Meets International Organization. In: European Journal of International Relations 9, H. 4, S. 543590 . 